\title{
FLS-Based Nonuniform Trajectory Tracking AILC for Uncertain Nonlinear Systems with Nonsymmetric Dead-Zone Input and Initial State Error
}

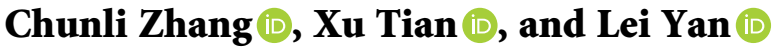 \\ Xi'an University of Technology, Shannxi Key Laboratory of Complex System Control and Intelligent Information Processing, \\ Xi'an, China \\ Correspondence should be addressed to Chunli Zhang; gaozhangchunli@163.com
}

Received 22 June 2021; Accepted 9 August 2021; Published 19 August 2021

Academic Editor: Xiao Chen

Copyright ( 92021 Chunli Zhang et al. This is an open access article distributed under the Creative Commons Attribution License, which permits unrestricted use, distribution, and reproduction in any medium, provided the original work is properly cited.

\begin{abstract}
This paper proposes an AILC method for uncertain nonlinear system to solve different target tracking problems. The method uses fuzzy logic systems (FLS) to approximate every uncertain term in systems. All closed-loop signals are bounded on $[0, T]$ according to the Lyapunov theory. A time-varying boundary layer and a typical convergent series are introduced to handle initial state error, unknown bounds of errors, and nonuniform target tracking, respectively. The result is that the tracking error's norm can converge to a small neighborhood along iteration increasing asymptotically. Finally, the simulation results of mass-spring mechanical system show the correctness of the theory and validity of the method.
\end{abstract}

\section{Introduction}

The research of the nonuniform trajectory is an interesting problem. The paper [1] proposed a new ILC law for firstorder hybrid parametric system and the paper [2] proposed a novel AILC method for nonlinear hybrid parameter systems. Recently, AILC is presented; a nonuniform target tracking AILC method was proposed in the paper [3]. The paper [4] proposed a fault-tolerant ILC technique for mobile robots' nonrepetitive target tracking with output constraints. From the above literature analysis, solving the nonuniform target tracking problem for uncertain mechanical nonlinear system is an important problem.

Adaptive control is used to handle system's control problem about uncertainties which is a challenging problem. Adaptive control schemes learn uncertainties by adaptive laws. NN and FLS are used in the method as function approximators, such as the papers [5-8]. The literature [9] could complete the varying control tasks by designing an adaptive fuzzy ILC for uncertain nonlinear system. Based on RBF neural network approximation, the literature [10] proposed AILC for nonlinear pure-feedback systems to solve the nonuniform target tracking problem. The uniform AILC frame for uncertain nonlinear system was proposed in the paper [11]; by Lyapunov theory, it can prove the convergence. It should be noted that Lyapunov function-based AILC plays an important role in dealing with the timevarying parameter in the literatures $[9,11,12]$. But initial state error is a challenging one as they need to converge to zero for keeping stability. Only the papers $[9,10,13]$ considered this problem recently it is an important problem for AILC.

Due to the physical limitations of actuators, in real systems, control inputs are often constrained, such as dead-zone inputs. However, these constraints may damage the performance of the system. In the papers [14-23], control performance could be changed by using different techniques recently. The question of adaptive stabilization for time-delay system had been solved in the literature [16]. The paper [17] developed an adaptive backstepping method of uncertain nonlinear systems about nonsymmetric dead-zone. As yet, there is no report from the literature for the AILC of nonlinear systems with nonsymmetric dead-zone input and initial state error. This is a problem that needs to be solved urgently. 
In this paper, the nonuniform trajectory tracking issue is discussed for the uncertain nonlinear systems with nonsymmetric dead-zone input and initial state error. The contributions of the proposed control method are presented as follows:

(i) The nonuniform trajectory tracking issue is studied for uncertain nonlinear systems under nonsymmetric dead-zone input and initial state error issues.

(ii) The AILC method is used to uncertain nonlinear systems. FLS is introduced to learn unknown dynamic. A convergence order is introduced to solve the unknown bound and nonuniform target tracking problem.

Finally, simulation results of mass-spring mechanical system are given to verify the validity of the designed controller.

The remainder of this paper is organized as follows: in Section 2, the system description and related concepts are given in detail. The controller design process and main results are presented in Section 3. A simulation is shown in Section 4 . Section 5 is the conclusion.

\section{System Description}

2.1. System Model. The following nonlinear systems are considered:

$$
\begin{aligned}
\dot{x}_{1, k} & =x_{2, k}+f_{1}\left(\bar{x}_{1, k}\right), \\
\dot{x}_{j, k} & =x_{j+1, k}+f_{j}\left(\bar{x}_{j, k}\right), \\
\dot{x}_{n, k} & =\Gamma\left(u_{k}\right)+f_{n}\left(\bar{x}_{n, k}\right), \\
y_{k} & =x_{1, k},
\end{aligned}
$$

where $\bar{x}_{j, k}=\left[x_{1, k}, \ldots, x_{j, k}\right]^{T} \in R^{j}, x=\bar{x}_{n}$ is the state that is measured; $\Gamma\left(u_{k}\right) \in R$ represents the actuator characteristics and $y_{k} \in R$ is the system output; $f_{j}\left(\bar{x}_{j, k}\right), j=1,2, \ldots, n$, are smooth unknown nonlinear functions.

Here, the function $\Gamma\left(u_{k}\right)$ represents actuator output with nonsymmetric dead-zone and can be expressed as

$$
\Gamma\left(u_{k}\right)= \begin{cases}m_{r}\left(u_{k}-b_{r}\right), & \text { if } u_{k} \geq b_{r}, \\ 0, & \text { if }-b_{l}<u_{k}<b_{r}, \\ m_{l}\left(u_{k}+b_{l}\right), & \text { if } u_{k} \leq-b_{l},\end{cases}
$$

where $m_{r}$ and $m_{l}$ present the right and left slopes, respectively; $b_{r}$ and $b_{l}$ present the breakpoint of dead-zone. The nonsymmetric dead-zone can be rewritten as a combination of a line and a disturbance-like term [18].

$$
\Gamma\left(u_{k}\right)=m(t) u_{k}+d(t),
$$

where

$$
m(t)=\left\{\begin{array}{ll}
m, & \text { if } u_{k} \leq 0, \\
m_{r}, & \text { if } u_{k}>0,
\end{array} d(t)= \begin{cases}-m_{r} b_{r}, & \text { if } u_{k} \geq b_{r}, \\
-m(t) u_{k}, & \text { if }-b_{l}<u_{k}<b_{r}, \\
m_{l} b_{l}, & \text { if } u_{k} \leq-b_{l} .\end{cases}\right.
$$

Assumption 1. Parameters $m_{l}, m_{r}, b_{l}$, and $b_{r}$ are uncertain constants. There exists an unknown $v$ satisfing $0<v \leq \min \left\{m_{l}, m_{r}\right\}$, which is small enough. The unknown constant $\bar{d}$ is the upper bound of $d(t)$.

Designing an AILC law $u_{k}(t)$ on $[0, T]$ to make the output $y_{k}(t)$ following the target trajectory $y_{r, k}(t)$ is our control objective, that is to say, $\lim _{k \rightarrow \infty}\left\|y_{k}(t)-y_{r, k}(t)\right\| \leq \varrho$, where $\varrho$ is the small positive error. Guarantee that the closed-loop signals are bounded. $y_{r, k}(t)$ is the smooth desired target. $k$ is the iteration index.

2.2. Convergent Series Sequence. The following definition and lemma can be used in the design process.

Definition 1 (see [20]). $\left\{\Delta_{k}\right\}$ is a series convergent sequence which is shown as

$$
\Delta_{k}=\frac{a}{k^{l}}
$$

where $k=1,2, \ldots ; a$ and $l$ are the needed parameters, satisfing $a>0 \in R, l \geq 2 \in N$.

Lemma 1 (see [20]). For $\left\{1 / k^{l}\right\}$, where $k=1,2, \ldots, l \geq 2$, the inequality as follows holds:

$$
\lim _{k \rightarrow \infty} \sum_{j=1}^{k} \frac{1}{j^{l}} \leq 2 .
$$

2.3. Description of Fuzzy Logic System (FLS). The FLS has a good approximation property [24]. For a smooth $f(x) \in R$ can be approximated by

$$
f(x)=\left(W^{*}\right)^{T} S(x)+\delta(x),
$$

where $\delta(x)$ is the fuzzy approximated error, $\left(W^{*}\right)^{T}$ is the optimal weight vector satisfing

$$
\left(W^{*}\right)^{T}=\underset{W^{*} \in \Omega_{W^{*}}}{\arg \min }\left\{\sup _{x \in D_{x}}|\widehat{f}(x \mid W)-f(x)|\right\},
$$

where $D_{x}$ represents the set of $x$ and $\hat{f}(x \mid W)$ that is estimated $f$, shown by

$$
\widehat{f}(x \mid W)=W^{T} S(x),
$$

with an adjustable $W$, and $\Omega_{W^{*}}$ is given as follows:

$$
\Omega_{W^{*}}=\left\{W^{*} \mid\left\|W^{*}\right\| \leq W_{M}^{*}\right\},
$$

with $W_{M}^{*}$ being a positive constant.

Assumption 2. In this paper, the following inherent approximation error $\delta_{j}\left(\bar{x}_{j, k}\right)$ is assumed to be bounded with $\left|\delta_{j}\left(\bar{x}_{j, k}\right)\right| \leq \theta_{j}$, where the unknown parameters $\theta_{j}(1 \leq j \leq n)$ denote the smallest upper bounds of $\left|\delta_{j}\left(\bar{x}_{j, k}\right)\right|$ with $\theta_{j} \geq 0$. 
2.4. Time-Varying Boundary Layer. Introduce the function $z_{j \phi, k}$ as follows to deal with initial state errors:

$$
\begin{aligned}
z_{j \phi, k} & =z_{j, k}-\phi_{j, k}(t) \operatorname{sat}\left(\frac{z_{j, k}}{\phi_{j, k}(t)}\right), \\
\phi_{j, k}(t) & =\varepsilon_{j, k} e^{-\eta t},
\end{aligned}
$$

where $z_{j, k}$ and $z_{j \phi, k}$ are variables of $t ; \varepsilon_{j, k}$ is a series convergence sequence; the saturation function sat is given as

$$
\operatorname{sat}\left(\frac{z_{j, k}}{\phi_{j, k}(t)}\right)= \begin{cases}1, & \text { if } z_{j, k}>\phi_{j, k}(t), \\ \frac{z_{j, k}}{\phi_{j, k}(t)}, & \text { if }-\phi_{j, k}(t) \leq z_{j, k} \leq \phi_{j, k}(t), \\ -1, & \text { if } z_{j, k}<-\phi_{j, k}(t),\end{cases}
$$

where $\phi_{j, k}(t)$ is the time-varying boundary layer. The control objective can be achieved by this. See the literature [9] for specific analysis.

Assumption 3. The initial state errors must satisfy $\left|z_{j, k}(0)\right|=$ $\varepsilon_{j, k}$ for some positive parameters $\varepsilon_{j, k}$ which are known; $j=1, \ldots, m, z_{j, k}(0)$ are given later in this paper.

\section{AILC Design and Convergence Analysis}

The specific process about designing controller is given as follows.

Step 1. Let $z_{1, k}=x_{1, k}-y_{r, k}, \quad z_{2, k}=x_{2, k}-\alpha_{1, k}$, $F_{1}\left(\bar{x}_{1, k}\right)=f_{1}\left(\bar{x}_{1, k}\right)$, and $\alpha_{1, k}$ is a virtual controller. Introduce the error function $z_{1 \phi, k}$ and $z_{2 \phi, k}$ from Section 2.4 to deal with initial state errors as

$$
\begin{aligned}
z_{1 \phi, k} & =z_{1, k}-\phi_{1, k}(t) \operatorname{sat}\left(\frac{z_{1, k}}{\phi_{1, k}(t)}\right), \\
\phi_{1, k}(t) & =\varepsilon_{1, k} e^{-\eta_{1} t}, \\
z_{2 \phi, k} & =z_{2, k}-\phi_{2, k}(t) \operatorname{sat}\left(\frac{z_{2, k}}{\phi_{2, k}(t)}\right), \\
\phi_{2, k}(t) & =\varepsilon_{2, k} e^{-\eta_{2} t} .
\end{aligned}
$$

Recall that

$$
\dot{x}_{1, k}=x_{2, k}+F_{1}\left(\bar{x}_{1, k}\right) \text {. }
$$

Derivate $z_{1 \phi, k}$ as follows:

$$
\dot{z}_{1 \phi, k}=z_{2, k}+\alpha_{1, k}+F_{1}\left(\bar{x}_{1, k}\right)-\dot{y}_{r, k}-\operatorname{sgn}\left(z_{1 \phi, k}\right) \dot{\phi}_{1, k} \text {. }
$$

According to Section 2.3, by FLS, $F_{1}\left(\bar{x}_{1, k}\right)$ is approximated as follows:

$$
F_{1}\left(\bar{x}_{1, k}\right)=W_{1}^{T} S_{1}\left(\bar{x}_{1, k}\right)+\delta_{1}\left(\bar{x}_{1, k}\right),
$$

where $\delta_{1}\left(\bar{x}_{1, k}\right)$ is the approximation error and $W_{1}$ is the optimal weight vector.

Define $N_{1}=\omega_{M 1}^{2}$, which is needed later; $\Delta_{k}=\left(a / k^{l}\right)$, $a>0$, and $l \geq 2$. The virtual controller is taken as

$$
\alpha_{1, k}=-\widehat{W}_{1, k}^{T} S_{1}\left(\bar{x}_{1, k}\right)-\widehat{N}_{1, k} \frac{1}{\Delta_{k}} z_{1 \phi, k}+\dot{y}_{r, k}-\eta_{1} z_{1, k} .
$$

Substitute equations (16) and (17) into equation (15), then

$$
\begin{aligned}
\dot{z}_{1 \phi, k}= & z_{2, k}-\widehat{N}_{1, k} \frac{1}{\Delta_{k}} z_{1 \phi, k} \\
& +W_{1}^{T} S_{1}\left(\bar{x}_{1, k}\right)+\delta_{1}\left(\bar{x}_{1, k}\right)-\widehat{W}_{1, k}^{T} S_{1}\left(\bar{x}_{1, k}\right) \\
& -\eta_{1} z_{1, k}-\operatorname{sgn}\left(z_{1 \phi, k}\right) \dot{\phi}_{1, k}(t) \\
= & z_{2 \phi, k}-\widehat{N}_{1, k} \frac{1}{\Delta_{k}} z_{1 \phi, k}-\widetilde{W}_{1, k}^{T} S_{1}\left(\bar{x}_{1, k}\right)+\delta_{1}\left(\bar{x}_{1, k}\right) \\
& +\phi_{2, k}(t) \operatorname{sat}\left(\frac{z_{2, k}}{\phi_{2, k}(t)}\right)-\eta_{1} z_{1, k}-\operatorname{sgn}\left(z_{1 \phi, k}\right) \dot{\phi}_{1, k}(t),
\end{aligned}
$$

where the estimated $W_{1}$ and $N_{1}$ are $\widehat{W}_{1, k}$ and $\widehat{N}_{1, k}$, respectively. $\tilde{W}_{1, k}=\widehat{W}_{1, k}-W_{1}$ and $\tilde{N}_{1, k}=\widehat{N}_{1, k}-N_{1}$ are the errors of estimated parameters. The last two terms of equation (18) can be changed as

$$
\begin{aligned}
-\eta_{1} z_{1, k}- & \operatorname{sgn}\left(z_{1 \phi, k}\right) \dot{\phi}_{1, k}(t)=-\eta_{1} z_{1 \phi, k} \\
& -\eta_{1} \phi_{1, k}(t) \operatorname{sat}\left(\frac{z_{1, k}}{\phi_{1, k}(t)}\right)-\operatorname{sgn}\left(z_{1 \phi, k}\right) \dot{\phi}_{1, k}(t) \\
= & -\eta_{1} z_{1 \phi, k} .
\end{aligned}
$$

Equations (18) and (19) can be rewritten as

$$
\begin{aligned}
\dot{z}_{1 \phi, k}= & z_{2 \phi, k}-\widehat{N}_{1, k} \frac{1}{\Delta_{k}} z_{1 \phi, k}-\eta_{1} z_{1 \phi, k}+\widetilde{W}_{1}^{T} S_{1}\left(\bar{x}_{1, k}\right) \\
& +\delta_{1}\left(\bar{x}_{1, k}\right)+\phi_{2, k}(t) \operatorname{sat}\left(\frac{z_{2, k}}{\phi_{2, k}(t)}\right) .
\end{aligned}
$$

Let $\omega_{1}=\delta_{1}\left(\bar{x}_{1, k}\right)+\phi_{2, k}(t) \operatorname{sat}\left(z_{2, k}(t) / \phi_{2, k}(t)\right), \quad$ then equation (20) becomes

$$
\dot{z}_{1 \phi, k}=z_{2 \phi, k}-\widehat{N}_{1, k} \frac{1}{\Delta_{k}} z_{1 \phi, k}-\eta_{1} z_{1 \phi, k}+\widetilde{W}_{1}^{T} S_{1}\left(\bar{x}_{1, k}\right)+\omega_{1} .
$$

Assumption 4. The bounded term $\omega_{1}$ satisfies $\left|\omega_{1}\right| \leq \omega_{M 1}$, where $\omega_{M 1}$ is a positive parameter.

Take the following nonnegative function:

$$
V_{1, k}=\frac{1}{2} z_{1 \phi, k}^{2}+\frac{1}{2} \tilde{W}_{1, k}^{T} \Gamma_{11}^{-1} \tilde{W}_{1, k}+\frac{1}{2} \Gamma_{21}^{-1} \tilde{N}_{1, k}^{2},
$$


where $\Gamma_{11}$ and $\Gamma_{21}$ are symmetric matrices and are positive. Derivate $V_{1, k}$ according to equation (21), then

$$
\begin{aligned}
\dot{V}_{1, k}= & z_{1 \phi, k} z_{2 \phi, k}-\eta_{1} z_{1 \phi, k}^{2} \\
& -\widetilde{W}_{1, k}^{T} \Gamma_{11}^{-1}\left(\Gamma_{11} S_{1}\left(\bar{x}_{1, k}\right) z_{1 \phi, k}-\dot{\hat{W}}_{1, k}\right) \\
& -\widehat{N}_{1, k} \frac{1}{\Delta_{k}} z_{1 \phi, k}^{2}+\omega_{1, k} z_{1 \phi, k}+\Gamma_{21}^{-1} \widetilde{N}_{1, k} \dot{\hat{N}}_{1, k} \\
\leq & z_{1 \phi, k} z_{2 \phi, k}-\eta_{1} z_{1 \phi, k}^{2} \\
& -\tilde{W}_{1, k}^{T} \Gamma_{11}^{-1}\left(\Gamma_{11} S_{1}\left(\bar{x}_{1, k}\right) z_{1 \phi, k}-\dot{\hat{W}}_{1, k}\right) \\
& -\widehat{N}_{1, k} \frac{1}{\Delta_{k}} z_{1 \phi, k}^{2}+\frac{1}{\Delta_{k}} \omega_{M 1}^{2} z_{1 \phi, k}^{2}+\frac{1}{4} \Delta_{k}+\Gamma_{21}^{-1} \tilde{N}_{1, k} \dot{\hat{N}}_{1, k} \\
= & z_{1 \phi, k} z_{2 \phi, k}-\eta_{1} z_{1 \phi, k}^{2} \\
& -\tilde{W}_{1, k}^{T} \Gamma_{11}^{-1}\left(\Gamma_{11} S_{1}\left(\bar{x}_{1, k}\right) z_{1 \phi, k}-\dot{\hat{W}}_{1, k}\right) \\
& -\tilde{N}_{1, k} \Gamma_{21}^{-1}\left(\Gamma_{21} \frac{1}{\Delta_{k}} z_{1 \phi, k}^{2}-\dot{\hat{N}}_{1, k}\right)+\frac{1}{4} \Delta_{k} .
\end{aligned}
$$

In the previous equation, $m n \leq(1 / r) m^{2}+(1 / 4) n^{2} r$ is used, where $r=\Delta_{k}$.

Step j. $(2 \leq j \leq n-1)$. Denote $F_{j}\left(\bar{x}_{j, k}\right)=f_{j}\left(\bar{x}_{j, k}\right)-\sum_{l=1}^{j-1}$ $\left(\partial \alpha_{j-1, k} / \partial x_{l, k}\right) \dot{x}_{l, k}\left(\bar{x}_{l, k}\right), \quad N_{j}=\omega_{M j}^{2}$, which is given later. $z_{j+1, k}=x_{j+1, k}-\alpha_{j, k}$, the same to Step 1; $z_{j \phi, k}$ and $z_{(j+1) \phi, k}$ from Section 2.4 are introduced as

$$
\begin{aligned}
z_{j \phi, k} & =z_{j, k}-\phi_{j, k}(t) \operatorname{sat}\left(\frac{z_{j, k}}{\phi_{j, k}(t)}\right), \\
\phi_{j, k}(t) & =\varepsilon_{j, k} e^{-\eta_{j} t}, \\
z_{(j+1) \phi, k} & =z_{(j+1), k}-\phi_{(j+1), k}(t) \operatorname{sat}\left(\frac{z_{(j+1), k}}{\phi_{(j+1), k}(t)}\right), \\
\phi_{(j+1), k}(t) & =\varepsilon_{(j+1), k} e^{-\eta_{(j+1)} t} .
\end{aligned}
$$

Derivate $z_{j \phi, k}$ as follows:

$$
\dot{z}_{j \phi, k}=z_{j+1, k}+\alpha_{j, k}+f_{j}\left(\bar{x}_{j, k}\right)-\dot{\alpha}_{j-1, k}-\operatorname{sgn}\left(z_{j \phi, k}(t)\right) \dot{\phi}_{j, k},
$$

where $\quad \dot{\alpha}_{j-1, k}=\sum_{l=1}^{j-1}\left(\partial \alpha_{j-1, k} / \partial x_{l, k}\right)\left(x_{l+1, k}+f_{l}\left(\bar{x}_{l, k}\right)\right)+$ $\left(\partial \alpha_{j-1, k} / \partial \widehat{W}_{j-1, k}\right) \dot{\hat{W}}_{j-1, k}+\left(\partial \alpha_{j-1, k} / \partial \widehat{N}_{j-1, k}\right) \dot{\hat{N}}_{j-1, k}+\left(\alpha_{j-1, k}\right.$ $/ \partial t)$. Denote $\quad F_{j}\left(\bar{x}_{j, k}\right)=f_{j}\left(\bar{x}_{j, k}\right)-\sum_{l=1}^{j}\left(\partial \alpha_{j-1, k} / \partial x_{j, k}\right)$ $\left(x_{j+1, k}+f_{j}\left(\bar{x}_{j, k}\right)\right), \quad P_{j-1, k}=\left(\partial \alpha_{j-1, k} / \partial \widehat{W}_{j-1, k}\right) \dot{\hat{W}}_{j-1, k}+$ $\left(\partial \alpha_{j-1, k} / \partial \widehat{N}_{j-1, k}\right) \dot{\hat{N}}_{j-1, k}+\left(\alpha_{j-1, k} / \partial t\right)$, then equation (25) can be rewritten as

$$
\dot{z}_{j \phi, k}=z_{j+1, k}+\alpha_{j, k}+F_{j}\left(\bar{x}_{j, k}\right)-P_{j-1, k}-\operatorname{sgn}\left(z_{j \phi, k}(t)\right) \dot{\phi}_{j, k} .
$$

According to Section 2.3, $F_{j}\left(\bar{x}_{j, k}\right)$ by FLS can become

$$
F_{j}\left(\bar{x}_{j, k}\right)=W_{j}^{T} S_{j}\left(\bar{x}_{j, k}\right)+\delta_{j}\left(\bar{x}_{j, k}\right),
$$

where $\delta_{j}\left(\bar{x}_{j, k}\right)$ is the approximation error and $W_{j}$ is the optimal weight vector.

The virtual controller is taken as

$$
\alpha_{j, k}=-z_{(j-1) \phi, k}-\widehat{W}_{j, k}^{T} S_{j}\left(\bar{x}_{j, k}\right)-\widehat{N}_{j, k} \frac{1}{\Delta_{k}} z_{j \phi, k}+P_{j-1, k}-\eta_{j} z_{j, k} .
$$

Equations (27) and (28) are substituted into equation (26), then we have

$$
\begin{aligned}
\dot{z}_{j \phi, k}= & z_{j+1, k}-z_{(j-1) \phi, k}-\widehat{N}_{j, k} \frac{1}{\Delta_{k}} z_{j \phi, k}-\eta_{j} z_{j, k}-\operatorname{sgn}\left(z_{j \phi, k}(t)\right) \dot{\phi}_{j, k} \\
& +W_{j}^{T} S_{j}\left(\bar{x}_{j, k}\right)+\delta_{j}\left(\bar{x}_{j, k}\right)-\widehat{W}_{j, k}^{T} S_{j}\left(\bar{x}_{j, k}\right) \\
= & z_{j+1, k}-z_{(j-1) \phi, k}-\widehat{N}_{j, k} \frac{1}{\Delta_{k}} z_{j \phi, k}-\widetilde{W}_{j, k}^{T} S_{j}\left(\bar{x}_{j, k}\right) \\
& +\delta_{j}\left(\bar{x}_{j, k}\right)-\eta_{j} z_{j, k}-\operatorname{sgn}\left(z_{j \phi, k}\right) \dot{\phi}_{j, k} \\
= & z_{(j+1) \phi, k}-z_{(j-1) \phi, k}-\widehat{N}_{j, k} \frac{1}{\Delta_{k}} z_{j \phi, k}-\widetilde{W}_{j, k}^{T} S_{j}\left(\bar{x}_{j, k}\right) \\
& +\delta_{j}\left(\bar{x}_{j, k}\right)+\phi_{(j+1) \phi, k}(t) \operatorname{sat}\left(\frac{z_{(j+1) \phi, k}}{\phi_{(j+1), k}(t)}\right) \\
& -\eta_{j} z_{j, k}-\operatorname{sgn}\left(z_{j \phi, k}(t)\right) \dot{\phi}_{j, k},
\end{aligned}
$$

where $\widehat{W}_{j, k}$ and $\widehat{N}_{j, k}$ are the estimated parameters of $W_{j}$ and $N_{j}$, respectively. $\widehat{W}_{j, k}=\widehat{W}_{j, k}-W_{j}$ and $\widetilde{N}_{j, k}=\widehat{N}_{j, k}-N_{j}$ are estimated parameter errors. The last two terms of equation (30) can be changed as

$$
\begin{aligned}
-\eta_{j} z_{j, k}-\operatorname{sgn}\left(z_{j \phi, k}\right) \dot{\phi}_{j, k}(t)= & -\eta_{j} z_{j \phi, k}-\eta_{j} \phi_{j, k}(t) \operatorname{sat}\left(\frac{z_{j, k}}{\phi_{j, k}(t)}\right) \\
& -\operatorname{sgn}\left(z_{j \phi, k}\right) \dot{\phi}_{j, k}(t) \\
= & -\eta_{j} z_{j \phi, k} .
\end{aligned}
$$

Let $\quad \omega_{j}=\delta_{j}\left(\bar{x}_{j, k}\right)+\phi_{(j+1) \phi, k}(t) \operatorname{sat}\left(z_{(j+1) \phi, k} / \phi_{(j+1), k}(t)\right)$, then equation (29) becomes

$$
\begin{aligned}
\dot{z}_{j \phi, k}= & z_{(j+1) \phi, k}-z_{(j-1) \phi, k}-\eta_{j} z_{j \phi, k} \\
& -\widehat{N}_{j, k} \frac{1}{\Delta_{k}} z_{j \phi, k}+\widetilde{W}_{j, k}^{T} S_{j}\left(\bar{x}_{j, k}\right)+\omega_{j} .
\end{aligned}
$$

Assumption $j . \omega_{j}$ satisfies $\left|\omega_{j}\right| \leq \omega_{M j}$; here, $\omega_{M j}$ is unknown. Positive definite function is chosen: 


$$
V_{j, k}=V_{j-1, k}+\frac{1}{2} z_{j \phi, k}^{2}+\frac{1}{2} \widetilde{W}_{j, k}^{T} \Gamma_{1 j}^{-1} \tilde{W}_{j, k}+\frac{1}{2} \Gamma_{2 j}^{-1} \widetilde{N}_{j, k}^{2}
$$

Derivate $V_{j, k}$ according to equation (31),

$$
\begin{aligned}
\dot{V}_{j, k} \leq & z_{j \phi, k} z_{(j+1) \phi, k}-\sum_{l=1}^{j} \eta_{l} z_{l, k}^{2}-\sum_{l=1}^{j} \tilde{W}_{l, k}^{T} \Gamma_{1 l}^{-1}\left(\Gamma_{1 l} S_{j}\left(\bar{x}_{l, k}\right) z_{l \phi, k}-\dot{\widehat{W}}_{l, k}\right) \\
& -\sum_{l=1}^{j} \tilde{N}_{l, k} \Gamma_{2 l}^{-1}\left(\Gamma_{2 l} \frac{1}{\Delta_{k}} z_{l \phi, k}^{2}-\dot{\hat{N}}_{l, k}\right)+j \frac{1}{4} \Delta_{k} .
\end{aligned}
$$

Step $n$. Define $z_{n, k}=x_{n, k}-\alpha_{n-1, k} ; z_{n \phi, k}$ from Section 2.4 is introduced as

$$
\begin{aligned}
z_{n \phi, k} & =z_{n, k}-\phi_{n, k}(t) \operatorname{sat}\left(\frac{z_{n, k}}{\phi_{n, k}(t)}\right), \\
\phi_{n, k}(t) & =\varepsilon_{n, k} e^{-\eta_{n} t} .
\end{aligned}
$$

Derivate $z_{n \phi, k}$ as follows:

$$
\dot{z}_{n \phi, k}=\Gamma\left(u_{k}\right)+f\left(\bar{x}_{n, k}\right)-\dot{\alpha}_{n-1, k}-\operatorname{sgn}\left(z_{n \phi, k}\right) \dot{\phi}_{n, k}(t),
$$

where $\quad \dot{\alpha}_{n-1, k}=\sum_{l=1}^{n-1}\left(\partial \alpha_{n-1,} \quad k / \partial x_{l, k}\right)\left(x_{l+1, k}+f_{l}\left(\bar{x}_{l, k}\right)\right)+$ $\left(\partial \alpha_{n-1, k} / \partial \widehat{W}_{n-1, k}\right) \dot{\hat{W}}_{n-1, k}+\left(\partial \alpha_{n-1, k} / \partial \widehat{N}_{n-1, k}\right) \dot{\hat{N}}_{n-1, k}+\left(\alpha_{n-1, k}\right.$ $/ \partial t)$. Denote $F_{n}\left(\bar{x}_{n, k}\right)=f_{n}\left(\bar{x}_{n, k}\right)-\sum_{l=1}^{n}\left(\partial \alpha_{n-1, k} / \partial x_{l, k}\right)$ $\left(x_{l+1, k}+f_{l}\left(\bar{x}_{l, k}\right)\right), \quad P_{n-1, k}=\left(\partial \alpha_{n-1, k} / \partial \widehat{W}_{n-1, k}\right) \dot{\hat{W}}_{n-1, k}+$ $\left(\partial \alpha_{n-1, k} / \partial \hat{N}_{n-1, k}\right) \dot{\hat{N}}_{n-1, k}+\left(\alpha_{n-1, k} / \partial t\right)$, and by equation (3), then equation (35) can be rewritten as

$$
\dot{z}_{n \phi, k}=m(t) u_{k}+F\left(\bar{x}_{n, k}\right)-P_{n-1, k}-\operatorname{sgn}\left(z_{n \phi, k}\right) \dot{\phi}_{n, k}(t)+d(t),
$$

from which $F_{n}\left(\bar{x}_{n, k}\right)$ can be rewritten as follows:

$$
F_{n}\left(\bar{x}_{n, k}\right)=W_{n}^{T} S_{n}\left(\bar{x}_{n, k}\right)+\delta_{n}\left(\bar{x}_{n, k}\right),
$$

where $\delta_{n}\left(\bar{x}_{n, k}\right)$ is the approximation error and $W_{n}$ is an optimal weight vector.

Let $u_{1, k}=-z_{(n-1) \phi, k}-\widehat{W}_{n, k}^{T} S_{n}\left(\bar{x}_{n, k}\right)-\widehat{N}_{n, k}\left(1 / \Delta_{k}\right) z_{n, k}+$ $P_{n-1, k}-\eta_{n} z_{n, k}$. Take the actual controller as

$$
u_{k}=u_{1, k}+u_{2, k}
$$

where $u_{2, k}$ is designed to compensate for unknown input gain $m(t)$.

Then, according to equations (37) and (38), equation (36) can be rewritten as

$$
\begin{aligned}
\dot{z}_{n \phi, k}= & u_{k}+(m(t)-1) u_{k}+W_{n}^{T} S_{n}\left(\bar{x}_{n, k}\right)+\delta_{n}\left(\bar{x}_{n, k}\right) \\
& -P_{n-1, k}-\operatorname{sgn}\left(z_{n \phi, k}\right) \dot{\phi}_{n, k}(t)+d(t) \\
= & -z_{(n-1) \phi, k}-\widetilde{W}_{n, k}^{T} S_{n}\left(\bar{x}_{n, k}\right)-\widehat{N}_{n, k} \frac{1}{\Delta_{k}} z_{n, k} \\
& +m(t) u_{2, k}+(m(t)-1) u_{1, k} \\
& +\delta_{n}\left(\bar{x}_{n, k}\right)+d(t)-\eta_{n} z_{n, k}-\operatorname{sgn}\left(z_{n \phi, k}\right) \dot{\phi}_{n, k}(t),
\end{aligned}
$$

where $\widehat{W}_{n, k}$ and $\widehat{N}_{n, k}$ are the estimated parameters of $W_{n}$ and $N_{n}$, respectively. $\widehat{W}_{n, k}=\widehat{W}_{n, k}-W_{n}$ and $\widetilde{N}_{n, k}=\widehat{N}_{n, k}-N_{n}$ are the estimated parameter errors.

The last two terms of equation (39) can be changed as

$$
\begin{aligned}
-\eta_{n} z_{n, k}-\operatorname{sgn}\left(z_{n \phi, k}\right) \dot{\phi}_{n, k}(t)= & -\eta_{n} z_{n \phi, k}-\eta_{n} \phi_{n, k}(t) \operatorname{sat}\left(\frac{z_{n, k}}{\phi_{n, k}(t)}\right) \\
& -\operatorname{sgn}\left(z_{n \phi, k}\right) \dot{\phi}_{n, k}(t) \\
= & -\eta_{n} z_{n \phi, k} .
\end{aligned}
$$

Then,

$$
\begin{aligned}
\dot{z}_{n \phi, k}= & -z_{(n-1) \phi, k}-\eta_{n} z_{n \phi, k}-\widetilde{W}_{n, k}^{T} S_{n}\left(\bar{x}_{n, k}\right)-\widehat{N}_{n, k} \frac{1}{\Delta_{k}} z_{n, k} \\
& +m(t) u_{2, k}+(m(t)-1) u_{1, k}+\delta_{n}\left(\bar{x}_{n, k}\right)+d(t) .
\end{aligned}
$$

Take $\quad u_{2, k}=-\operatorname{sat}\left(z_{n, k} / \phi_{n, k}(t)\right)\left(\hat{\rho}_{k}(t)+1\right)\left|u_{1, k}\right|, \quad$ where $\hat{\rho}_{k}(t)$ is the estimation of uncertain parameter $\rho=1 / m(t)$ and let $\omega_{n}=\delta_{n}\left(\bar{x}_{n, k}\right)+d(t)$, then equation (41) becomes

$$
\begin{aligned}
\dot{z}_{n \phi, k}= & -z_{(n-1) \phi, k}-\eta_{n} z_{n \phi, k}-\tilde{W}_{n, k}^{T} S_{n}\left(\bar{x}_{n, k}\right)-\widehat{N}_{n, k} \frac{1}{\Delta_{k}} z_{n \phi, k} \\
& -m(t) \operatorname{sat}\left(\frac{z_{n, k}}{\phi_{n, k}(t)}\right)\left(\hat{\rho}_{k}(t)+1\right)\left|u_{1, k}\right|+(m(t)-1) u_{1, k}+\omega_{n} .
\end{aligned}
$$

Assumption $n$. Because of assumptions 1 and 2, $\omega_{n}$ is bounded such that $\left|\omega_{n}\right| \leq \omega_{M n}$ with $\omega_{M n}$ being unknown.

Adaptive learning laws are taken as follows:

$$
\begin{aligned}
\dot{\hat{W}}_{j, k} & =\Gamma_{1 j} S_{j}\left(\bar{x}_{j, k}\right) z_{j \phi, k}, \quad j=1, \ldots, m, \\
\dot{\hat{N}}_{j, k} & =\Gamma_{2 j} \frac{1}{\Delta_{k}} z_{j \phi, k}^{2}, \quad j=1, \ldots, m, \\
\dot{\hat{\rho}}_{k}(t) & =\Gamma_{3}\left|u_{1, k}\right|\left|z_{n \phi, k}\right|,
\end{aligned}
$$

where $\Gamma_{3}$ is the learning gain to be designed.

Positive definite function is chosen:

$$
V_{n, k}=V_{n-1, k}+\frac{1}{2} z_{n \phi, k}^{2}+\frac{1}{2} \widetilde{W}_{n, k}^{T} \Gamma_{1 n}^{-1} \widetilde{W}_{n, k}+\frac{1}{2} \Gamma_{2 n}^{-1} \widetilde{N}_{n, k}^{2}+\frac{1}{2} \Gamma_{3}^{-1} m(t) \widetilde{\rho}_{k}^{2} .
$$

Derivate $V_{n, k}$ according to equation (42) and substitute equations (43)-(45) into it, then

$$
\begin{aligned}
\dot{V}_{n, k} \leq & z_{(n-1) \phi, k} z_{n \phi, k}-\sum_{l=1}^{n-1} \eta_{l} z_{l \phi, k}^{2} \\
& -\sum_{l=1}^{n-1} \tilde{W}_{l, k}^{T} \Gamma_{1 l}^{-1}\left(\Gamma_{1 l} S_{l}\left(\bar{x}_{l, k}\right) z_{l \phi, k}-\dot{\hat{W}}_{l, k}\right)
\end{aligned}
$$




$$
\begin{aligned}
& -\sum_{l=1}^{n-1} \tilde{N}_{l, k} \Gamma_{2 l}^{-1}\left(\Gamma_{2 l} \frac{1}{\Delta_{k}} z_{l \phi, k}^{2}-\dot{\hat{N}}_{l, k}\right)+(n-1) \frac{1}{4} \Delta_{k} \\
& +z_{n \phi, k} \dot{z}_{n \phi, k}+\tilde{W}_{n, k}^{T} \Gamma_{1 n}^{-1} \dot{\hat{W}}_{n, k} \\
& +\Gamma_{2 n}^{-1} \tilde{N}_{n, k} \dot{\hat{N}}_{n, k}+\Gamma_{3}^{-1} m(t) \tilde{\rho}_{k} \dot{\hat{\rho}}_{k} \\
\leq & -\sum_{l=1}^{n} \eta_{l} z_{l \phi, k}^{2}-\sum_{l=1}^{n} \tilde{W}_{l, k}^{T} \Gamma_{1 l}^{-1}\left(\Gamma_{1 l} S_{l}\left(\bar{x}_{l, k}\right) z_{l \phi, k}-\dot{\hat{W}}_{l, k}\right) \\
& -\sum_{l=1}^{n-1} \tilde{N}_{l, k} \Gamma_{2 l}^{-1}\left(\Gamma_{2 l} \frac{1}{\Delta_{k}} z_{l \phi, k}^{2}-\dot{\hat{N}}_{l, k}\right)+(n-1) \frac{1}{4} \Delta_{k} \\
& -\widehat{N}_{n, k} \frac{1}{\Delta_{k}} z_{n \phi, k}^{2}-m(t) \widehat{\rho}_{k}(t)\left|u_{l, k}\right|\left|z_{n \phi, k}\right|+\left|u_{1, k}\right|\left|z_{n \phi, k}\right| \\
& +\frac{1}{\Delta_{k}} \omega_{N n}^{2} z_{n \phi, k}^{2}+\frac{1}{4} \Delta_{k}+\Gamma_{2 n}^{-1} \tilde{N}_{n, k} \dot{\hat{\hat{N}}}_{n, k}+\Gamma_{3}^{-1} m(t) \tilde{\rho}_{k} \dot{\hat{\rho}}_{k} \\
= & -\sum_{l=1}^{n} \eta_{l} z_{l \phi, k}^{2}-\sum_{l=1}^{n} \tilde{W}_{l, k}^{T} \Gamma_{1 l}^{-1}\left(\Gamma_{1 l} S_{l}\left(\bar{x}_{l, k}\right) z_{l \phi, k}-\dot{\hat{W}}_{l, k}\right) \\
& -\sum_{l=1}^{n} \tilde{N}_{l, k} \Gamma_{2 l}^{-1}\left(\Gamma_{2 l} \frac{1}{\Delta_{k}} z_{l \phi, k}^{2}-\dot{\hat{N}}_{l, k}\right)+n \frac{1}{4} \Delta_{k} \\
& -\Gamma_{3}^{-1} m(t) \tilde{\rho}_{k}\left(\left|\Gamma_{3}\left\|u_{1, k}\right\| z_{n \phi, k}\right|-\dot{\hat{\rho}}_{k}\right) \\
= & -\sum_{l=1}^{n} \eta_{l} z_{l, k}^{2}+n \frac{1}{4} \Delta_{k} .
\end{aligned}
$$

The assumption below is needed.

Assumption 5. For any $k$, as $t=0, \widehat{N}_{j, k}(0)=\widehat{N}_{j, k-1}(T)$, $\widehat{W}_{j, k}(0)=\widehat{W}_{j, k-1}(T) ; j=1, \ldots, m, \hat{\rho}_{k}(0)=\widehat{\rho}_{k-1}(T)$.

Theorem 1. Under assumptions 1, 2, 3, 4-n, and 5, design controller equation (38), and adaptive parameter estimation laws (43)-(45) for system (1), we get all signals bounded, and

$$
\lim _{k \rightarrow \infty} z_{l \phi, k}(t)=0, \quad l=1,2, \ldots, n,
$$

i.e., $\lim _{k \longrightarrow \infty} z_{1 \phi, k}(t)=0,\left\|z_{1, k}(t)\right\|<\phi_{1, \infty}(t)+$ s as $k \longrightarrow \infty$, where $\varsigma$ is a small positive parameter.

Proof. From Section 2.4, $\left\|z_{\phi, k}(0)\right\|^{2}=0 \leq\left\|z_{\phi, k}(T)\right\|^{2}$, where $z_{\phi, k}=\left[z_{1 \phi, k}, z_{2 \phi, k}, \ldots, z_{n \phi, k}\right]^{T}$. By (46), we get

$$
V_{n, k}\left(z_{\phi, k}(0), \widehat{W}_{k}(T), \widehat{N}_{k}(T), \widehat{\rho}_{k}(T)\right) \leq V_{n, k}(0)+\int_{0}^{T} \dot{V}_{n, k} \mathrm{~d} t,
$$

$\underset{\left.\ldots, \widehat{N}_{n, k}\right]^{T}}{\text { where }} \widehat{W}_{k}=\left[\widehat{W}_{1, k}, \widehat{W}_{2, k}, \ldots, \widehat{N}_{n, k}\right]^{T}, \quad \widehat{N}_{k}=\left[\widehat{N}_{1, k}, \widehat{N}_{2, k}\right.$, Substituting equation (47) into equation (49), we get

$$
\begin{gathered}
V_{n, k}\left(z_{\phi, k}(0), \widehat{W}_{k}(T), \widehat{N}_{k}(T), \hat{\rho}_{k}(T)\right) \leq V_{n, 1}(0) \\
-\sum_{j=1}^{k} \sum_{l=1}^{m} \int_{0}^{T} \eta_{l}\left(z_{l \phi, j}\right)^{2} \mathrm{~d} t+n\left(\frac{1}{4}\right) T\left(\sum_{j=1}^{k} \Delta_{j}\right) .
\end{gathered}
$$

Denote $V_{0, k}=V_{n, 1}(0)+n(1 / 4) T\left(\sum_{j=1}^{k} \Delta_{j}\right)$, then equation (50) can be rewritten as

$$
\sum_{l=1}^{k} \sum_{l=1}^{n} \int_{0}^{T} \eta_{l}\left(z_{l \phi, j}\right)^{2} \mathrm{~d} t \leq V_{0, k}-V_{n, k}\left(z_{\phi, k}(0), \widehat{W}_{k}(T), \widehat{N}_{k}(T), \widehat{\rho}_{k}(T)\right) .
$$

By equation (6), $\lim _{k \longrightarrow \infty} V_{0, k} \leq V_{n, 1}(0)+2$ an $(1 / 4) T$, then $V_{0, k}$ is bounded; by assumption 5 and equation (50), $V_{n, k}(0)$ can be bounded. $V_{n, k}\left(z_{k}(0), \widehat{W}_{k}(T), \widehat{N}_{k}(T)\right.$, $\left.\widehat{\rho}_{k}(T)\right) \geq 0$, so

$$
\lim _{k \rightarrow \infty} \sum_{l=1}^{n} \int_{0}^{T} \eta_{l}\left(z_{l \phi, k}\right)^{2} \mathrm{~d} t=0 .
$$

By equation (46), for any $k, \quad V_{n, k}(t)=V_{n, k}$ $(0)+\int_{0}^{t} \dot{V}_{n, k}(\tau) \mathrm{d} \tau$, by (47), then

$$
V_{n, k}(t) \leq V_{n, k}(0)-\sum_{l=1}^{n} \int_{0}^{t} \eta_{l}\left(z_{l \phi, k}(\tau)\right)^{2} \mathrm{~d} \tau+\operatorname{tn}\left(\frac{1}{4}\right) \Delta_{k} .
$$

By equation (52), $\sum_{l=1}^{n} \int_{0}^{t} \eta_{l}\left(z_{l \phi, k}(\tau)\right)^{2} \mathrm{~d} \tau$ is bounded. By Definition $1, \Delta_{k}$ is bounded, and $t \in[0, T]$, so $\operatorname{tn}(1 / 4) \Delta_{k}$ is also bounded. From above all, for any $k, V_{n, k}(t)$ is bounded, then we have $x_{j, k} ; \widehat{W}_{k}(t)$ and $\widehat{N}_{k}(t)$ are bounded. By equation (38), $u_{k}$ is bounded. By equation (31), $\dot{z}_{j \phi, k}$ is bounded; $z_{j \phi, k}$ is continuous uniformly; according to Barbalat lemma, $z_{j \phi, k} \longrightarrow 0$ when $k \longrightarrow \infty$, i.e., $k \longrightarrow \infty$, $z_{1 \phi, k} \longrightarrow 0$, then $\left\|z_{1 \phi, k}\right\|<\varsigma . \quad\left\|z_{1, k}\right\|=\| z_{1 \phi, k}+\operatorname{sgn}\left(z_{1 \phi, k}\right)$ $\phi_{1, k}(t)\|\leq\| z_{1 \phi, k}\|+\| \phi_{1, k}(t)\|<\| \phi_{1, k}(t) \|+\varsigma$, so $k \longrightarrow \infty$, $\left\|z_{1, k}\right\|<\phi_{1, \infty}(t)+\varsigma$, finished.

\section{Simulation}

In this section, a mass-spring mechanical system is considered to show the effectiveness of the proposed controller. $\bar{m}$ is a mass; assume that resistive force caused by friction is zero. The external force $u_{k}$ drives the mass, which is a control variable. $y_{k}$ is the displacement from a reference position; the motion equation of the system with nonsymmetric deadzone input is as follows:

$$
\bar{m} \ddot{y}_{k}+F_{m s}\left(y_{k}\right)=\Gamma\left(u_{k}\right),
$$

where $t \in[0, \pi], F_{m s}(\cdot)$ is the spring's restoring force; $k$ denotes the iteration index.

We define $x_{1, k}=y_{k}, x_{2, k}=\dot{y}_{k}$, and $\bar{m}=1$, so equation (54) can be transformed into the state-space form

$$
\begin{aligned}
& \dot{x}_{1, k}=x_{2, k}, \\
& \dot{x}_{2, k}=\Gamma\left(u_{k}\right)-F_{m s}\left(x_{1, k}\right) .
\end{aligned}
$$

The spring's restoring force can be modeled as

$$
F_{m s}\left(x_{1, k}\right)=k x_{1, k}\left(\sum_{i=0}^{q} a_{i} x_{1, k}^{i}\right) \text {. }
$$

In the system, we have $k=1, a_{0}=0, a_{1}=a_{2}=a_{3}=$ $a_{4}=1$, and $q=4$. 


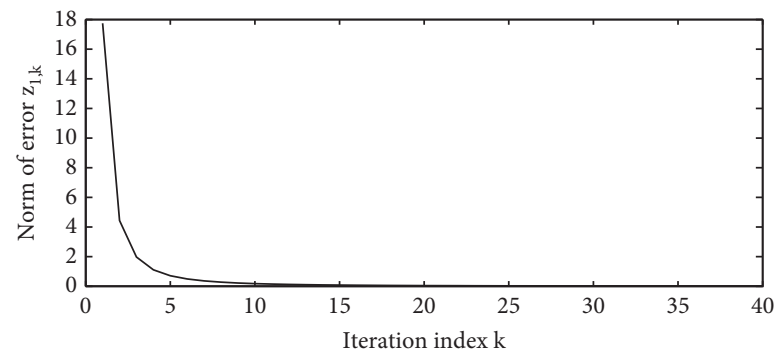

Figure 1: Curve graph of $\left\|z_{1, k}\right\|$ along $k$.

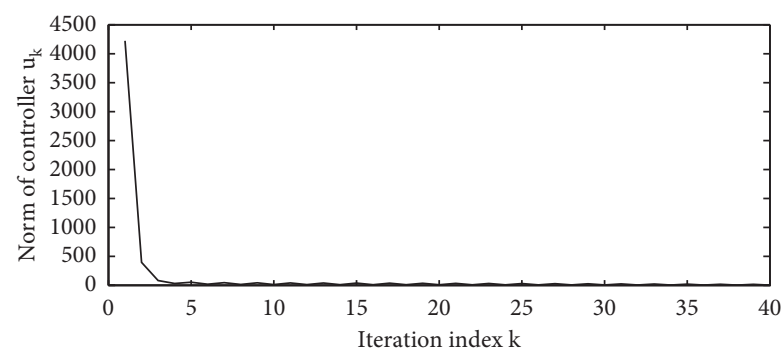

Figure 2: Curve graph of $\left\|u_{k}\right\|$ along $k$.
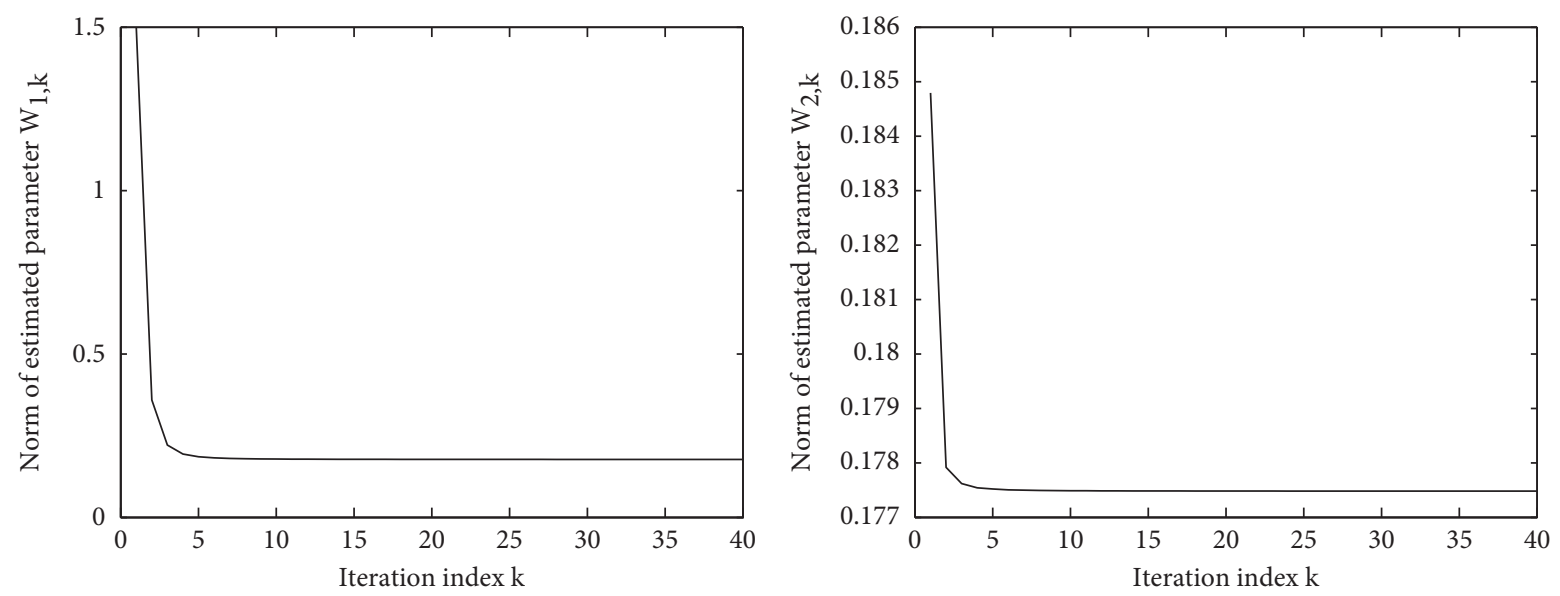

Figure 3: Curve graph of $\left\|\widehat{W}_{1, k}\right\|,\left\|\widehat{W}_{2, k}\right\|$ along $k$.
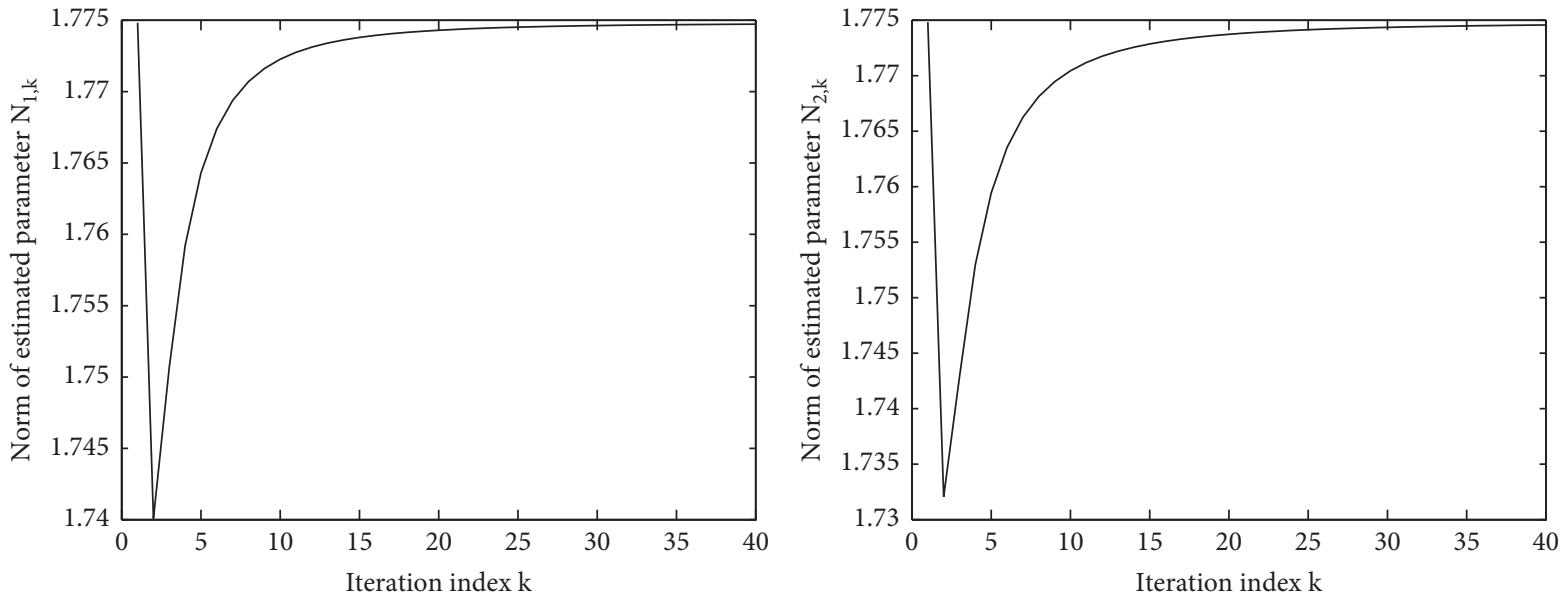

Figure 4: Curve graph of $\left\|\widehat{N}_{1, k}\right\|,\left\|\widehat{N}_{2, k}\right\|$ along $k$. 
The nonsymmetric dead-zone is shown as

$$
\Gamma\left(u_{k}\right)= \begin{cases}u_{k}-1, & \text { if } u_{k} \geq 1, \\ 0, & \text { if }-3<u_{k}<1, \\ u_{k}+3, & \text { if } u_{k} \leq-3 .\end{cases}
$$

System objective is that the output of system (54) can follow the reference trajectory $y_{r, k}$ on $[0, \pi]$ when $k \longrightarrow \infty$. In the different target case, $y_{r, k}=g_{k} \sin (2 t)$ as $k$ is even, and $y_{r, k}=g_{k} \cos (t)$ as $k$ is odd, where $g_{k}=\operatorname{rand}(0,1)$.

By Theorem 1, the AILC is chosen as

$$
\begin{aligned}
\alpha_{1, k} & =-\widehat{W}_{1, k}^{T} S_{1}\left(\bar{x}_{1, k}\right)-\widehat{N}_{1, k} \frac{1}{\Delta_{k}} z_{1 \phi, k}+\dot{y}_{r, k}-\eta_{1} z_{1, k}, \\
u_{k} & =u_{1, k}+u_{2, k}, \\
u_{1, k} & =-z_{1 \phi, k}-\widehat{W}_{2, k}^{T} S_{2}\left(\bar{x}_{2, k}\right)-\widehat{N}_{2, k} \frac{1}{\Delta_{k}} z_{2, k}+P_{1, k}-\eta_{2} z_{2, k}, \\
u_{2, k} & =-\operatorname{sat}\left(\frac{z_{2, k}}{\phi_{2, k}(t)}\right)\left(\hat{\rho}_{k}(t)+1\right)\left|u_{1, k}\right|,
\end{aligned}
$$

where $P_{1, k}=\left(\partial \alpha_{1, k} / \partial \widehat{W}_{1, k}\right) \dot{\widehat{W}}_{1, k}+\left(\partial \alpha_{1, k} / \partial \widehat{N}_{1, k}\right) \dot{\hat{N}}_{1, k}+\left(\alpha_{1, k}\right.$ $\left./ \partial y_{r}\right) \dot{y}_{r}+\left(\alpha_{1, k} / \partial \dot{y}_{r}\right) \ddot{y}_{r}$ and the estimation laws are represented by equations (43)-(45), where $\eta_{1}=10, \eta_{2}=1$, $\Delta_{k}=a / k^{2}, \quad a=100 / 3, \quad \Gamma_{11}=\operatorname{diag}\{0.1,0.1,0.1,0.1,0.1\}$, $\Gamma_{21}=1, \Gamma_{12}=\operatorname{diag}\{0.1,0.1,0.1,0.1,0.1\}, \Gamma_{22}=1$, and $\Gamma_{3}=1$. The inputs of the FLS are $x_{1, k}, x_{2, k}$; the membership function of every variable is given as follows:

$$
\begin{aligned}
& \mu_{A_{i}^{1}}=\exp \left(-\left(\frac{y_{i}+a_{i}}{b_{i}}\right)^{2}\right), \\
& \mu_{A_{i}^{2}}=\exp \left(-\left(\frac{y_{i}+0.5 \times a_{i}}{b_{i}}\right)^{2}\right), \\
& \mu_{A_{i}^{3}}=\exp \left(-\left(\frac{y_{i}}{b_{i}}\right)^{2}\right), \\
& \mu_{A_{i}^{4}}=\exp \left(-\left(\frac{y_{i}-0.5 \times a_{i}}{b_{i}}\right)^{2}\right), \\
& \mu_{A_{i}^{5}}=\exp \left(-\left(\frac{y_{i}-a_{i}}{b_{i}}\right)^{2}\right),
\end{aligned}
$$

where $i=1, \ldots, 4, \quad y_{1}=x_{1, k}, y_{2}=x_{2, k}, a_{1}=a_{2}=2$, and $b_{1}=b_{2}=2$, and the fuzzy rules are given as

$$
R_{t}^{j}: \text { if } y_{t} \text { is } A_{t}^{j}, f_{s}\left(\bar{x}_{s}\right) \text { is } B_{s}^{j},
$$

where $s=1,2$ : as $s=1, t=1,2,3$, as $s=2, t=1,2,4$. The period of sampling is $0.01 \mathrm{~s}$.

Here, choose the parameters and initial values as follows: $z_{1, k}(0)=1 / k^{2}, \quad z_{2, k}(0)=1 / k^{2}, \quad \widehat{W}_{1,1}(0)=[0.01,0,0,0,0]^{T}$, $\widehat{W}_{2,1}(0)=[0.01,0,0,0,0]^{T}, \widehat{N}_{1,1}(0)=-0.1, \widehat{N}_{2,1}(0)=-0.1$,

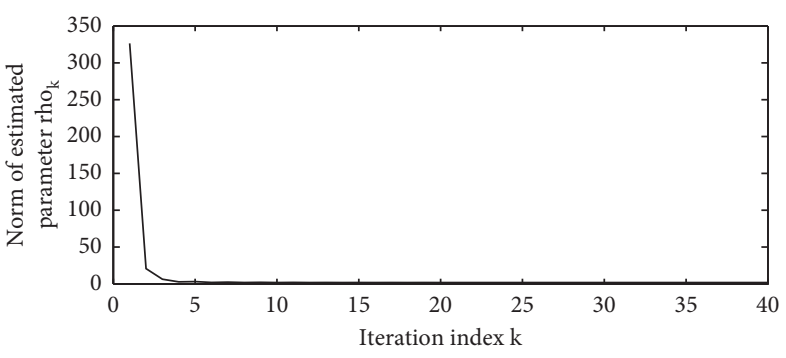

Figure 5: Curve graph of $\left\|\hat{\rho}_{k}\right\|$ along $k$.

and $\widehat{\rho}_{1}(0)=0.1$. Taking $k=40$, Figures $1-5$ are the simulation figures.

Figure 1 shows the convergence of error. Moreover, on the interval $[0, \pi]$, Figures $2-5$ give that $\left\|u_{k}\right\|,\left\|\widehat{W}_{1, k}\right\|,\left\|\widehat{W}_{2, k}\right\|$, $\left\|\widehat{N}_{1, k}\right\|,\left\|\widehat{N}_{2, k}\right\|$, and $\left\|\widehat{\rho}_{k}\right\|$ are bounded. Figures $1-5$ confirm the validity of control scheme which is developed in this article from the simulation results.

\section{Conclusions}

The different target tracking problem for unknown nonlinear systems with nonsymmetric dead-zone input is solved. Introduce FLS to deal with the uncertain dynamics, and the problem of approximation error and initial state error can be efficiently solved by suitable means. This paper can keep all signals being bounded on $[0, T]$; errors can converge to a small set along iteration increasing. Simulation study proves the correctness of AILC method of this paper.

\section{Data Availability}

No data were used to support this study.

\section{Conflicts of Interest}

The authors declare that they have no conflicts of interest.

\section{Acknowledgments}

This work was supported by the National Natural Science Foundation of China under Grant no. 61603296 and Natural Science Foundation Project of Shaanxi Provincial Education Department under Grant no. 16JK1541.

\section{References}

[1] J.-X. Xu and J. Xu, "On iterative learning from different tracking tasks in the presence of time-varying uncertainties," IEEE Transactions on Systems, Man and Cybernetics, Part B (Cybernetics), vol. 34, no. 1, pp. 589-597, 2004.

[2] J. M. Li, Y. P. Sun, and Y. Liu, "Hybrid adaptive iterative learning control of non-uniform trajectory tracking," Control Theory \& Applications, vol. 25, no. 1, pp. 100-104, 2008.

[3] C.-L. Zhang and J.-M. Li, “Adaptive iterative learning control of non-uniform trajectory tracking for strict feedback nonlinear time-varying systems with unknown control direction," Applied Mathematical Modelling, vol. 39, no. 10-11, pp. 2942-2950, 2015. 
[4] J. Xu, "Fault-tolerant iterative learning control for mobile robots non-repetitive trajectory tracking with output constraints," Automatica, vol. 94, pp. 63-71, 2018.

[5] H. Yue and S. Yu, "Adaptive fuzzy tracking control for a class of stochastic nonlinearly parameterized systems with distributed input delay," Journal of the Franklin Institute, vol. 353, no. 3, pp. 713-734, 2016.

[6] H. Y. Yue, J. R. Shi, L. Y. Du et al., "Adaptive fuzzy tracking control for a class of perturbed nonlinearly parameterized systems using minimal learning parameters algorithm," Iranian Journal of Fuzzy Systems, vol. 15, no. 3, pp. 99-116, 2018.

[7] H. Y. Yue et al., "Fuzzy adaptive tracking control for a class of nonlinearly parameterized systems with unknown control directions, Iran," Journal of Fuzzy Systems, vol. 16, no. 5, pp. 97-112, 2019

[8] H. Yue, Z. Wei, Q. Chen, and X. Zhang, "Dynamic surface control for a class of nonlinearly parameterized systems with input time delay using neural network," Journal of the Franklin Institute, vol. 357, no. 4, pp. 1961-1986, 2020.

[9] C. J. Chiang-Ju Chien, "A combined adaptive law for fuzzy iterative learning control of nonlinear systems with varying control tasks," IEEE Transactions on Fuzzy Systems, vol. 16, no. 1 , pp. $40-51,2008$.

[10] C. L. Zhang and X. Tian, "Non-uniform trajectory tracking adaptive iterative learning control for nonlinear pure-feedback systems with initial state error based on RBF-neural network," in Proceedings of the 40th Chinese Control Conference, Shanghai, China, July 2021.

[11] A. Taybi and C. J. Chien, "A unified adaptive iterative learning control framework for uncertain nonlinear systems," IEEE Transactions on Automatic Control, vol. 52, no. 10, pp. 1907-1913, 2007.

[12] J. X. Jian-Xin Xu and R. Rui Yan, "Adaptive learning control for finite interval tracking based on constructive function approximation and wavelet," IEEE Transactions on Neural Networks, vol. 22, no. 6, pp. 893-905, 2011.

[13] C. Zhang and J. Li, "Adaptive iterative learning control for nonlinear pure-feedback systems with initial state error based on fuzzy approximation," Journal of the Franklin Institute, vol. 351, no. 3, pp. 1483-1500, 2014.

[14] J.-X. Xu and R. Yan, "Iterative learning control design without a priori knowledge of the control direction," Automatica, vol. 40, no. 10, pp. 1803-1809, 2004.

[15] Y. Tan, H.-H. Dai, D. Huang, and J.-X. Xu, "Unified iterative learning control schemes for nonlinear dynamic systems with nonlinear input uncertainties," Automatica, vol. 48, no. 12, pp. 3173-3182, 2012.

[16] X. J. Wu, X. L. Wu, R. Zhen, X. y. Luo, and Q. M. Zhu, "Adaptive control for time-delay non-linear systems with non-symmetric input non-linearity," International Journal of Modelling, Identification and Control, vol. 13, no. 3, pp. 152-160, 2011.

[17] X. Luo, X. Guan, and X. Wu, “Adaptive backstepping faulttolerant control for unmatched non-linear systems against actuator dead-zone," IET Control Theory and Applications, vol. 4, no. 5, pp. 879-888, 2010.

[18] X. Wu, X. Wu, X. Luo, Q. Zhu, and X. Guan, "Neural network-based adaptive tracking control for nonlinearly parameterized systems with unknown input nonlinearities," Neurocomputing, vol. 82, pp. 127-142, 2012.

[19] W. S. Chen, "Adaptive backstepping dynamic surface control for systems with periodic disturbances using neural networks," IET Control Theory and Applications, vol. 3, no. 10, pp. 1383-1394, 2009.
[20] S. Zhu, M.-X. Sun, and X.-X. He, "Iterative learning control of strict-feedback nonlinear time-varying systems," Acta Automatica Sinica, vol. 36, no. 3, pp. 454-458, 2010.

[21] J. Li and J. Li, "Adaptive iterative learning control for coordination of second-order multi-agent systems," International Journal of Robust and Nonlinear Control, vol. 24, no. 18, pp. 3282-3299, 2014.

[22] Y. Chen, Z. Liu, C. L. P. Chen, and Y. Zhang, "Adaptive fuzzy control of switched nonlinear systems with uncertain deadzone: a mode-dependent fuzzy dead-zone model," Neurocomputing, vol. 432, pp. 133-144, 2021.

[23] D. Meng and K. L. Moore, "Learning to cooperate: networks of formation agents with switching topologies," Automatica, vol. 64 , pp. 278-293, 2016.

[24] L. X. Wang, "Fuzzy systems are universal approximators," in Proceedings of the IEEE International Conference on Fuzzy Systems, pp. 1163-1170, San Diego, CA, USA, March 1992. 